\title{
Prognóstico da hipoglicemia hiperinsulinêmica persistente da infância - uma revisão sistemática
}

\author{
Prognosis of persistent hyperinsulinemic hypoglycemia of infancy - a systematic review
}

\author{
Cláudio Flauzino Oliveira ${ }^{1}$, Mário Cícero Falcão
}

RESUMO

Objetivo: Descrever o prognóstico de crianças com hipoglicemia hiperinsulinêmica persistente da infância, submetidos ou não à pancreatectomia.

Fontes de dados: Foram selecionados, por meio das bases de dados Medline e Ovid, estudos publicados nos últimos dez anos contendo o termo "byperinsulinemic bypoglycemia”. A partir desta seleção, foram analisados os artigos que realizaram estudos observacionais sobre o prognóstico da hipoglicemia hiperinsulinêmica persistente da infância (zero a dez anos), para a revisão sistemática.

Síntese dos dados: Foram identificadas 269 publicações, das quais 13 continham informações sobre prognóstico das crianças com hipoglicemia hiperinsulinêmica persistente da infância, incluindo oito artigos referentes a pacientes submetidos à pancreatectomia. $\mathrm{O}$ principal determinante do prognóstico é o quadro neurológico. Nos estudos apresentados, a incidência de retardo do desenvolvimento neurológico variou de 10 a $70 \%$, dependendo da casuística e da idade de aparecimento dos sintomas. Entre as crianças submetidas à pancreatectomia para controle da hipoglicemia, o principal fator que influenciou o prognóstico em longo prazo foi a característica e a extensão da lesão pancreática. As lesões focais acarretam significativamente menos complicações que as difusas. A incidência de diabetes melito após a pancreatectomia parcial ou subtotal variou de 25 a $100 \%$ dos pacientes, de acordo com a casuística avaliada.

Conclusões: $\mathrm{O}$ momento de aparecimento dos sintomas influi diretamente sobre a gravidade do quadro neurológico e, consequentemente, sobre o prognóstico. Nos pacientes submetidos à pancreatectomia, a característica da lesão determina maiores ou menores conseqüências.

Palavras-chave: hipoglicemia; hiperinsulinismo; prognóstico.

\section{ABSTRACT}

Objective: To describe the prognosis of persistent hyperinsulinemic hypoglycemia of infancy, submitted or not to pancreatectomy.

Data sources: Databases Medline and Ovid were searched for studies published in the last ten years with the expression "hyperinsulinemic hypoglycemia". Manuscripts about the prognosis of patients with persistent hyperinsulinemic hypoglycemia of infancy (zero to ten years) were analyzed, in order to do a systematic review.

Data synthesis: Two hundred sixty-nine publications were identified, and 13 had information about the prognosis of the persistent hyperinsulinemic hypoglycemia of infancy, including eight studies of patients submitted to pancreatectomy. The main factor associated with the prognosis was the neurological status. In the analyzed studies, the incidence of neurological development delay varied from 10 to $70 \%$, depending on the sample and on the age of onset. Among children submitted to pancreatectomy in order to treat the hypoglycemia, the main factors that influenced prognosis were the characteristics and the extension of the pancreatic injury. Focal injuries caused significantly less complications that the diffuse injuries. The incidence of diabetes mellitus after a partial or subtotal pancreatectomy varied from 25 to $100 \%$, depending on the patients evaluated in each study.

Conclusions: The timing of symptoms onset influences directly the severity of the neurological status and the prognosis. In the patients submitted to pancreatectomy, the characteristics of the pancreatic injury determine the extent of the consequences.

Key-words: hypoglycemia; hyperinsulinism; prognosis.
'Pós-graduando do Departamento de Pediatria da Faculdade de Medicina da Universidade de São Paulo (FMUSP)

${ }^{2}$ Doutor em Pediatria pela FMUSP
Rua Vieira de Moraes, 45, apto. 51

CEP 04617-010 - São Paulo/SP

E-mail: profmariofalcao@yahoo.com.br

Recebido em: 12/3/2007

Aprovado em: 18/6/2007 


\section{Introdução}

A hipoglicemia hiperinsulinêmica persistente da infância é a causa mais comum de hipoglicemia recorrente e de difícil controle em recém-nascidos e lactentes. Este termo vem sendo utilizado nas publicações mais recentes, no lugar de nesidioblastose ou síndrome de dismaturidade das ilhotas pancreáticas.

A hipersecreção inapropriada de insulina é responsável por episódios de hipoglicemia de gravidade variável. Mutações genéticas têm sido descritas em diversos genes, principalmente no SUR1 e no Kir6.2, que afetam a função dos canais de potássio dependentes de ATP das células $\beta$ do pâncreas, podendo ter herança autossômica recessiva ou dominante. Além disso, há diferenciação entre lesões focais e difusas das células $\beta^{(1)}$.

Os pacientes com hipoglicemia hiperinsulinêmica persistente da infância são, na maioria das vezes, recém-nascidos grandes para a idade gestacional, que apresentam sintomas persistentes de hipoglicemia, incluindo convulsões de difícil controle, freqüentemente desde o período neonatal. A relação insulina/glicose alterada e a manutenção de níveis elevados de insulina na vigência de hipoglicemia são parâmetros importantes no diagnóstico. $\mathrm{O}$ tratamento do hiperinsulinismo inclui agentes que suprimem a secreção de insulina, que antagonizam seu efeito nos tecidos ou que reduzem as ilhotas. O tratamento cirúrgico tem indicação nos casos de insucesso da terapia clínica ou nos casos de hiperprodução focal de insulina ${ }^{(2)}$.

O prognóstico é muito variável e difícil de ser adequadamente determinado, uma vez que vários casos não são diagnosticados ou são identificados em fases diversas da evolução da doença. Além disso, a gravidade da hipoglicemia e a característica focal ou difusa da lesão pancreática influem no prognóstico. Pacientes submetidos à pancreatectomia também podem apresentar conseqüências e diferentes evoluções em longo prazo ${ }^{(1,2)}$.

O objetivo deste estudo é, por meio de uma revisão sistemática, descrever o prognóstico das crianças com hipoglicemia hiperinsulinêmica persistente da infância, submetidas ou não à pancreatectomia.

\section{Metodologia}

Foram selecionados, por meio das bases de dados Medline (www.pubmed.com) e Ovid (www.portaldapesquisa.com. br), estudos publicados nos últimos dez anos, em inglês ou em português, contendo, em qualquer parte do texto, o termo "byperinsulinemic hypoglycemia". Artigos relevantes citados nas bibliografias dos textos selecionados foram igualmente revisados.

A partir dos resumos, foram escolhidos os artigos contendo estudos observacionais sobre prognóstico da hipoglicemia hiperinsulinêmica persistente da infância, incluindo pacientes submetidos a tratamento clínico ou cirúrgico. Foram também identificados e analisados artigos brasileiros, bem como relatos de caso com achados infreqüentes e não incluídos nos estudos observacionais.

Para a inclusão dos estudos, foi levado em consideração o grau de evidência de cada pesquisa, conforme descrito abaixo:

- A: grandes ensaios clínicos aleatorizados e meta-análises;

- B: estudos clínicos e observacionais delineados adequadamente;

- C: relatos e séries de casos clínicos;

- D: publicações baseadas em consensos e opiniões de especialistas.

\section{Resultados}

Foram identificadas 269 publicações, das quais 13 continham informações sobre prognóstico das crianças com hipoglicemia hiperinsulinêmica persistente da infância, incluindo oito artigos referentes a pacientes submetidos à pancreatectomia. A estes estudos, foram adicionados dois artigos brasileiros, na forma de relato de caso e de revisão da literatura.

\section{Estudos com tratamento clínico}

No maior estudo de coorte publicado até esta data, informações de 114 pacientes foram retiradas de um banco de dados alemão a respeito de hiperinsulinismo congênito, localizado em Düsseldorf (grau de evidência B). A análise do prognóstico mostrou que $27 \%$ dos pacientes com início neonatal da hipoglicemia desenvolveram aumento de peso (acima de dois desvios padrão), $44 \%$ dos pacientes apresentaram retardo psicomotor ou mental $-18 \%$ grave e $26 \%$ leve - e $25 \%$ tiverem epilepsia ${ }^{(3)}$.

O desenvolvimento neurológico de 90 pacientes com hipoglicemia hiperinsulinêmica persistente da infância foi retrospectivamente analisado em um estudo publicado em 2001 (grau de evidência B). Dos pacientes, 54 eram recém-nascidos, sendo oito tratados clinicamente e 46 submetidos à cirurgia (19 por lesão focal e 27 por lesão difusa). Retardo psicomotor grave foi encontrado em sete pacientes, dos quais seis eram neonatos; desabilidade psicomotora intermediária ocorreu em 12 pacientes e a epilepsia, em 16. Neste estudo, o início da sintomatologia no período neonatal foi o principal fator de risco para retardo neuropsicomotor grave e epilepsia ${ }^{(4)}$. 
Uma série de pacientes australianos, publicada em 2005 (grau de evidência C), revisou, durante o período de 16 anos, o desfecho clínico de 14 pacientes com hipoglicemia hiperinsulinêmica persistente da infância. O diagnóstico ocorreu, em média, no terceiro mês de vida; $71,4 \%$ apresentaram crises convulsivas, $64 \%$ tiveram atraso de desenvolvimento, que foi classificado como grave em $14,5 \%$ dos pacientes ${ }^{(5)}$.

Em 2002, uma análise retrospectiva de dez pacientes tailandeses (grau de evidência C) mostrou atraso de desenvolvimento em três (30\%) crianças, sendo que uma delas também evoluiu com epilepsia ${ }^{(6)}$.

Entre os achados pouco freqüentes, Camurdan et al ${ }^{(7)}$ relataram o caso de uma paciente de seis meses de idade com hipoglicemia hiperinsulinêmica persistente da infância (grau de evidência $\mathrm{C}$ ), em quem foi feito também o diagnóstico de síndrome de West, achado relatado apenas uma vez na literatura em associação com hiperamonemia. Em 2002, Nachum et al ${ }^{(8)}$ descreveram dois irmãos com hipoglicemia hiperinsulinêmica persistente da infância (grau de evidência C) e atrofia cerebral precoce. Um estudo indiano publicado em 1998 revelou a existência de infecções recorrentes graves em cerca de $30 \%$ dos pacientes com hipoglicemia hiperinsulinêmica persistente da infância (grau de evidência $C)^{(9)}$.

Em 2000, um grupo de São Paulo relatou o caso de gêmeos com hipoglicemia hiperinsulinêmica persistente da infância (grau de evidência C). Embora as duas irmãs já apresentassem episódios de hipoglicemia, o diagnóstico só ocorreu com três meses e meio de idade, após o aparecimento de crises convulsivas. Com o tratamento clínico, as duas irmãs evoluíram com controle da hipoglicemia e das crises convulsivas. Seguidas até quatro anos e nove meses, ambas apresentavam desenvolvimento neuropsicomotor adequado para a idade ${ }^{(10)}$.

Outra publicação brasileira de 2005 relatou o caso de um paciente com hipoglicemia desenvolvida no período neonatal (grau de evidência C). Tratada precocemente, a criança respondeu bem à terapia com reposição de glicose, octreotide e dexametasona. Em seguimento ambulatorial, não apresentava alteração do desenvolvimento neurológico até o momento da publicação ${ }^{(11)}$.

\section{Estudos com tratamento cirúrgico}

Basicamente, existem duas abordagens cirúrgicas: a pancreatectomia parcial, quando a lesão pancreática é focal, e a pancreatectomia sub-total, quando a lesão pancreática é difusa; nestes casos, geralmente retira-se $95 \%$ do pâncreas.

Em 2005, Cherian e Abduljabbar ${ }^{(12)}$ publicaram estudo avaliando o desenvolvimento de recém-nascidos e lactentes com hipoglicemia hiperinsulinêmica persistente da infância submetidos à extração cirúrgica de $95 \%$ do tecido pancreático. Os pacientes foram seguidos por um período de um a 20 anos, com média de 13,3 anos, e analisou-se: o crescimento e desenvolvimento, o controle glicêmico, o estado neurológico e desempenho escolar (grau de evidência B). As dez crianças incluídas no estudo desenvolveram diabetes melito, três das quais no período pós-operatório precoce. Com exceção de um paciente que havia apresentado hemorragia subaracnoídea, edema cerebral e crises convulsivas no período neonatal, todos os outros mostraram desenvolvimento neurológico normal. Nenhum evoluiu com manifestação clínica de insuficiência pancreática exócrina.

Em 1999, um estudo francês realizado com 52 recémnascidos com hiperinsulinismo tratados cirurgicamente determinou o prognóstico pós-operatório por meio de determinações da glicose plasmática e da hemoglobina glicosilada, além de testes de tolerância à glicose (grau de evidência B). Trinta e nove recém-nascidos tinham lesão difusa e foram tratados com pancreatectomia subtotal; 22 tinham lesões locais e foram submetidos à pancreatectomia parcial. Aqueles com lesões focais não apresentaram sinais de hipoglicemia e tinham valores normais de glicose plasmática pré- e pós-prandial, de hemoglobina glicosilada e do teste de tolerância à glicose. Por outro lado, após pancreatectomia subtotal, 13 dos 30 pacientes persistiram com hipoglicemia, oito desenvolveram diabetes melito e sete, hiperglicemia. Apenas dois destes pacientes com lesões difusas apresentavam níveis de glicose plasmática normais um ano após a cirurgia ${ }^{(13)}$.

Em uma série de pacientes australianos publicada em 2005, das quatro crianças submetidas à pancreatectomia, apenas uma desenvolveu diabetes melitos ${ }^{(5)}$. Em dois pacientes paquistaneses submetidos à pancreatectomia subtotal, nenhum apresentou diabetes melito; no entanto, um deles necessitou de suplemento de enzimas pancreáticas ${ }^{(14)}$.

Nos dados coletados a partir do banco de dados de Düsseldorf, $40 \%$ dos pacientes persistiram com hipoglicemia após a pancreatectomia, enquanto que, em $27 \%$, houve desenvolvimento de diabetes melito ${ }^{(3)}$.

Das dez crianças tailandesas relatadas em 2002, seis não puderam ser tratadas clinicamente e necessitaram de pancreatectomia subtotal. Destas, apenas uma apresentou diabetes melito e síndrome de má absorção ${ }^{(6)}$.

No relato de 45 pacientes submetidos à pancreatectomia parcial eletiva entre 1983 e 2000, todos possuíam a forma 
focal da hipoglicemia hiperinsulinêmica persistente da infância e foram operados guiados por dosagens de insulina realizadas na veia pancreática, de maneira a ressecar apenas a parte lesada do pâncreas (grau de evidência B). Com exceção de um paciente, todos se tornaram normoglicêmicos ${ }^{(15)}$.

Em uma série de dez casos publicada em 2000 (grau de evidência $\mathrm{C}$ ), as crianças submetidas à pancreatectomia foram analisadas em dois grupos, com base na idade de início da hipoglicemia: precoce ( $<1$ mês) ou tardia $(\geq 1$ mês). Dos sete pacientes do grupo de início precoce submetidos à pancreatectomia ( 85 a 95\% do tecido pancreático), três persistiram com hipoglicemia e dois precisaram ser reoperados, mas continuaram a apresentar hipoglicemia. Os outros quatro pacientes se tornaram normoglicêmicos. Apenas um deles desenvolveu diabetes melito seis anos após a cirurgia. Seis dos sete pacientes apresentaram atraso de desenvolvimento e coeficiente de inteligência abaixo do normal. As três crianças do grupo precoce foram submetidas à pancreatectomia e se tornaram normoglicêmicas ${ }^{(16)}$.

Em 1997, Shilyansky et a ${ }^{(17)}$ publicaram uma série de 27 crianças submetidas à pancreatectomia subtotal desde 1971 (grau de evidência C). Houve recorrência da hiperinsulinemia e da hipoglicemia em $33 \%$ das crianças e $56 \%$ desenvolveram diabetes melito.

\section{Discussão}

A hipoglicemia hiperinsulinêmica persistente da infância se manifesta geralmente no período neonatal ou durante o primeiro ano de vida. A apresentação clínica varia de acordo com a idade do início da hipoglicemia. Da mesma forma, o prognóstico também depende da idade de apresentação dos sintomas. Como observado na maioria dos estudos, o início da hipoglicemia no período neonatal é fator de risco para retardo grave do desenvolvimento neuropsicomotor e para o aparecimento de epilepsia.

Sem dúvida, o principal determinante do prognóstico dos pacientes com hipoglicemia hiperinsulinêmica persistente da infância é o quadro neurológico. Nos estudos apresentados, a incidência de retardo do desenvolvimento neurológico varia de cerca de 10 até quase $70 \%$, dependendo da casuística e da idade de aparecimento dos sintomas. Ainda com relação ao prognóstico neurológico, a ocorrência de epilepsia se assemelha ao retardo do desenvolvimento neuropsicomotor. No levantamento de literatura realizado, a freqüência observada nos estudos também foi de 10 a $71 \%$.

Além destes dois achados comumente relatados e que, por si só, já justificam uma grande preocupação com esta doença, há descrição da ocorrência de síndrome de West em associação com hipoglicemia hiperinsulinêmica persistente da infância, coexistindo ou não com hiperamonemia. A documentação de pacientes com atrofia cerebral desde o período antenatal, relatada em dois casos israelenses, explica, em parte, os motivos pelos quais existe elevada incidência de desenvolvimento neurológico insatisfatório. Por outro lado, este achado de atrofia levanta a hipótese de que os danos neurológicos não ocorram tão somente pela hipoglicemia, uma vez que tais pacientes já apresentavam atrofia cerebral antes mesmo de desenvolverem hipoglicemia.

Menos freqüentes que as conseqüências neurológicas na hipoglicemia hiperinsulinêmica persistente da infância, outros achados completam o prognóstico, como a ocorrência de sobrepeso em algumas casuísticas e o encontro, não reproduzido em outros estudos, de infecções graves recorrentes.

Entre as crianças submetidas à pancreatectomia para controle da hipoglicemia, o principal fator que influencia o prognóstico em longo prazo é a característica da lesão pancreática. $\mathrm{O}$ manejo das lesões focais acarreta significativamente menos complicações que o tratamento das lesões difusas. Em algumas casuísticas de pacientes apenas com lesão focal, não há praticamente nenhuma ocorrência de distúrbio glicêmico futuro, especialmente se forem adotadas técnicas que tornem ainda mais precisa a excisão cirúrgica, como a dosagem intra-operatória da insulina na veia pancreática. Nestes pacientes, tampouco se observou o desenvolvimento de insuficiência pancreática exócrina.

Entretanto, o prognóstico é mais complicado nos pacientes com lesão pancreática difusa. Nestes, em que a pancreatectomia pode ser parcial ou subtotal, chegando até a 95 ou 99\% do total do volume do pâncreas, há incidência tanto de hipoglicemia persistente como de diabetes melito e insuficiência exócrina.

Nos estudos analisados, a incidência de diabetes melito após pancreatectomia parcial ou subtotal variou de 25 a $100 \%$ dos pacientes, de acordo com a casuística avaliada. Naqueles em que a ressecção não foi suficientemente ampla, houve ocorrência de hipoglicemia persistente, acometendo entre 20 e $48 \%$ das crianças estudadas.

Pelo exposto, a hipoglicemia hiperinsulinêmica persistente da infância é importante pela atenção que requer para o seu diagnóstico. O momento de aparecimento dos sintomas influi diretamente na gravidade do quadro e no prognóstico, assim como, em pacientes submetidos à pancreatectomia, a característica da lesão determina maiores ou menores conseqüências. O fato de, na maioria das vezes, o prognóstico ser determinado por alterações neurológicas faz com que especial atenção deva ser conferida a esta grave afecção. 


\section{Referências bibliográficas}

1. Sperling MA, Menon RK. Differential diagnosis and management of neonatal hypoglycemia. Pediatr Clin North Am 2004;51:703-23.

2. Dekelbab BH, Sperling MA. Hyperinsulinemic hypoglycemia of infancy: the challenge continues. Diabetes Metab Res Rev 2004;20:189-95.

3. Meissner T, Wendel U, Burgard P, Schaetzle S, Mayatepek E. Long-term follow-up of 114 patients with congenital hyperinsulinism. Eur J Endocrinol 2003;149:43-51.

4. Menni F, de Lonlay P, Sevin C, Touati G, Peigné C, Barbier V et al. Neurologic outcomes of 90 neonates and infants with persistent hyperinsulinemic hypoglycemia. Pediatrics 2001;107:476-9.

5. Ismail D, Werther G. Persistent hyperinsulinaemic hypoglycaemia of infancy: 15 years' experience at the Royal Children's Hospital $(\mathrm{RCH})$, Melbourne. J Pediatr Endocrinol Metab 2005;18:1103-9.

6. Sawathiparnich P, Likitmaskul S, Angsusingha K, Nimkarn S, Chaichanwatanakul K, Laohapansang $\mathrm{M}$ et al. Persistent hyperinsulinemic hypoglycemia of infancy: experience at Siriraj Hospital. J Med Assoc Thai 2002;85:S506-12.

7. Camurdan MO, Cinaz P, Serdaroglu A, Bideci A, Demirel F. Persistent hyperinsulinemic hypoglycemia presenting with a rare complication: West syndrome. J Pediatr Endocrinol Metab 2004;17:1465-8.

8. Nachum Z, Ben-Shlomo I, Rakover Y, Weiner E, Shalev E. Early brain atrophy in persistent hyperinsulinemic hypoglycemia of infancy. J Pediatr 2002;141:706-9.

9. Desai MP, Khatri JV. Persistent hyperinsulinemic hypoglycemia of infancy. Indian Pediatr 1998;35:317-28
10. Lazarine DF, Pahl MM, Damiani D, Dichtchekenian V, Setian N, Okay Y. Hipoglicemia hiperinsulinêmica persistente da infância em gêmeos: relato de caso. J Pediatr (Rio J) 2000;76:162-8.

11. Ferraz DP, Almeida MA, Mello BF. Uso de octreotide na hipoglicemia hiperinsulinêmica persistente infantil. Arq Bras Endocrinol Metab 2005;49:460-7.

12. Cherian MP, Abduljabbar MA. Persistent hyperinsulinemic hypoglycemia of infancy (PHHI): long-term outcome following 95\% pancreatectomy. J Pediatr Endocrinol Metab 2005;18:1441-8.

13. de Lonlay-Debeney P, Poggi-Travert F, Fournet JC, Sempoux C, Vici CD, Brunelle $\mathrm{F}$ et al. Clinical features of 52 neonates with hyperinsulinism. N Engl J Med 1999;340:1169-75.

14. Aslam M, Safdar CA, Khalid A, Awan S, Ahmed I, Ahmed Z. Persistent hyperinsulinemic hypoglycemia of infancy - nesidioblastosis. J Coll Physicians Surg Pak 2004;14:501-3.

15. Cretolle C, Fekete CN, Jan D, Nassogne MC, Saudubray JM, Brunelle F et al. Partial elective pancreatectomy is curative in focal form of permanent hyperinsulinemic hypoglycaemia in infancy: A report of 45 cases from 1983 to 2000. J Pediatr Surg 2002;37:155-8.

16. Mahachoklertwattana P, Suprasongsin C, Teeraratkul S, Preeyasombat C. Persistent hyperinsulinemic hypoglycemia of infancy: long-term outcome following subtotal pancreatectomy. J Pediatr Endocrinol Metab 2000;13:37-44.

17. Shilyansky J, Fisher S, Cutz E, Perlman K, Filler RM. Is $95 \%$ pancreatectomy the procedure of choice for treatment of persistent hyperinsulinemic hypoglycemia of the neonate? J Pediatr Surg 1997;32:342-6. 